\title{
Second breast conserving therapy after ipsilateral breast tumor recurrence - a 10-year experience of re-irradiation
}

\author{
Tobias Forster, MD 1,2,3, Sati Akbaba, MD 1,2,3, Daniela Schmitt, Dr. rer. nat.,2,3, David Krug, MD',4, Rami El Shafie, MD 1,2,3, \\ Jan Oelmann-Avendano, MD1,2,3, Prof. Katja Lindel, MD5, Laila König, MD'1,2,3, Nathalie Arians, MD1,2,3, \\ Denise Bernhardt, MD',2,3 Prof. Frederik Marmé, MD6,7. Prof. Andreas Schneeweiss, MD 3,7. Prof. Jörg Heil, MD6,7, \\ Prof. Christof Sohn, MD6. Prof. Jürgen Debus, MD, PhD, Dr. rer. nat.12,3,8,9,10, Juliane Hörner-Rieber, PD, MD1,2,3,8 \\ 'Department of Radiation Oncology, Heidelberg University Hospital, Heidelberg, Germany, ${ }^{2}$ Heidelberg Institute of Radiation Oncology \\ (HIRO), Heidelberg, Germany, ${ }^{3}$ National Center for Tumor Diseases (NCT), Heidelberg, Germany, ${ }^{4}$ Department of Radiation Oncology \\ University Hospital Schleswig-Holstein, Kiel, Germany, ${ }^{5}$ Department of Radiation Oncology, Municipal Hospital, Karlsruhe, Germany, \\ 'Department of Gynecology and Obstetrics, Heidelberg University Hospital, Heidelberg, Germany, '7 German Cancer Research Center \\ (DKFZ), Heidelberg, Germany, ${ }^{8}$ Clinical Cooperation Unit Radiation Oncology, German Cancer Research Center (DKFZ), Heidelberg, \\ Germany, 'Heidelberg lon-Beam Therapy Center (HIT), Department of Radiation Oncology, Heidelberg University Hospital, Heidelberg, \\ Germany. ${ }^{10}$ German Cancer Consortium (DKTK), partner site Heidelberg, Germany
}

\begin{abstract}
Purpose: The aim of this study is to evaluate the efficacy and toxicity of post-operative partial breast re-irradiation with multi-catheter brachytherapy after second breast conserving therapy (BCT) in patients with small, low-risk ipsilateral breast tumor recurrence (IBTR).

Material and methods: Between 2008 and 2018, 19 consecutive patients with low-risk IBTR (max. rpT1 cN0 cM0, Her2 negative, preferably positive hormone receptor status) who refused mastectomy were treated with salvage lumpectomy, followed by post-operative partial breast re-irradiation with multi-catheter brachytherapy. Eight patients were irradiated using PDR brachytherapy (49.8-50.4 Gy in pulses of 0.5-0.7 Gy) and 11 patients using HDR brachytherapy (34.2 Gy in fractions of $3.8 \mathrm{~Gy}$ or $32 \mathrm{~Gy}$ in fractions of $4 \mathrm{~Gy}$ ). All patients had undergone prior BCT for their primary tumor, followed by adjuvant whole breast radiotherapy. Local control (LC), locoregional control (LRC), overall survival (OS), disease-free survival (DFS) as well as toxicity were evaluated in the present study.

Results: After a median follow-up of 65 months following IBTR (18-120 months), only one second IBTR in 19 patients was diagnosed 77 months after re-irradiation, resulting in a LC rate of $100 \%$ at 5 years. DFS and OS rates were both $100 \%$ at 5 years following re-irradiation. Except for the above mentioned second IBTR, no regional or distant relapse was recorded. Regarding toxicity, $63 \%$ of patients developed adverse events (CTCAE grade $\leq 2$ ), with fibrosis detected in $37 \%(7 / 19)$ of patients, necrosis in $11 \%$ (2/19), hyperpigmentation in $47 \%(9 / 19)$, and telangiectasia in $11 \%$ $(2 / 19)$, respectively. No patient showed a high-grade (CTCAE grade $\geq 3$ ) adverse event.

Conclusions: In case of small, low-risk IBTR, adjuvant re-irradiation using multi-catheter brachytherapy is a feasible, safe, and effective treatment method after repeated lumpectomy, and an alternative to mastectomy.

J Contemp Brachytherapy 2019; 11, 4: 312-319 DOI: https://doi.org/10.5114/jcb.2019.87001
\end{abstract}

Key words: breast cancer, re-irradiation, brachytherapy, second breast conserving therapy, ipsilateral breast tumor recurrence.

\section{Purpose}

Breast cancer is the second most common cancer in the world and, by far, the most frequent cancer among women, with an estimated 1.67 million new cancer cases diagnosed in 2012 (25\% of all cancers) [1]. Ipsilateral breast tumor recurrence (IBTR) after surgery and adju- vant therapy is recorded in up to $20 \%$ of patients within 15 years after initial therapy [2]. Several prognostic factors influencing the risk of IBTR have been reported including age, tumor characteristics inherited susceptibility, treatment concepts of the primary tumor, and lifestyle factors like obesity and alcohol consumption [3]. Howev-
Address for correspondence: Juliane Hörner-Rieber, PD, MD, Department of Radiation Oncology, Received: 10.04 .2019 Heidelberg University, Im Neuenheimer Feld 400, 69120 Heidelberg, Germany, phone: +49 6221566999 , e-mail: juliane.hoerner-rieber@med.uni-heidelberg.de
Accepted: 31.05.2019

Published: 29.08.2019 
er, early-stage low-risk tumors with favorable prognostic characteristics are known to show a significantly smaller risk for local tumor recurrence [4,5], with 5-year rates of only one to $2 \%$ [6].

Initially, standard therapy for women with all tumor stages was mastectomy. In the early 1980's, breast conserving therapy (BCT) was established for small, low-risk tumors, combining lumpectomy plus adjuvant radiation therapy [7]. Nevertheless, if local breast tumor recurrence is diagnosed after previous whole-breast radiotherapy, mastectomy still remains the gold-standard therapy according to current national and international guidelines. However, several studies reported high levels of psychosocial distress negatively affecting quality of life in some women undergoing mastectomy [8]. Hence, some women refuse this form of radical, mutilating surgery and request for alternative treatment options. Some authors therefore considered the possibility of a second BCT with salvage lumpectomy as sole therapy for small local recurrences with favorable tumor characteristics [9]. However, significantly increased second IBTR rates of up to $20 \%$ after 5 years have been reported for lumpectomy alone $[10,11,12,13]$. Consequently, the addition of post-operative re-irradiation following lumpectomy was proposed to reduce the rates of second IBTR [3,14,15,16,17,18,19,20,21]. As studies reporting outcomes following re-irradiation with local brachytherapy for women with small, low-risk IBTR treated with second lumpectomy are still rare, the efficacy and toxicity of this individual treatment concept were evaluated at the Department of Radiooncology at the Heidelberg University Hospital.

\section{Material and methods}

In total, 19 patients with IBTR were re-irradiated using multi-catheter brachytherapy at the Heidelberg University Hospital between January 2008 and September 2018. All patients received a breast-conserving surgery for the primary tumor with sentinel lymph node biopsy (5 patients) or axillary lymph node dissection (12 patients), followed by whole breast external beam radiation therapy (EBRT) with or without regional nodal irradiation, depending on the pathological axillary lymph node status. Whole breast irradiation of the primary tumor was performed with a total dose of $50.4 \mathrm{~Gy}$ in fractions of 1.8 Gy and an additional boost irradiation of 8-16 Gy was performed in 16 patients. One patient received additional irradiation of the supraclavicular lymphatic drainage, with the same total dose and number of fractions. Systematic adjuvant treatment was administered according to guidelines. Six patients received adjuvant chemotherapy: one patient was treated with epirubicin and cyclophosphamide, two patients received cyclophosphamide, methotrexate and 5-fluorouracil, while one patient was treated with mitoxantrone. Furthermore, epirubicin, cyclophosphamide, and 5-fluorouracil were administered in two patients. Fourteen patients received endocrine therapies according to their hormone receptor status. Endocrine therapy consisted of tamoxifen in 13 cases, while one patient was treated with an aromatase inhibitor. Pa- tients were followed up according to German and international treatment guidelines.

Ipsilateral breast tumor recurrence was defined as local recurrence within the pre-treated breast. All patients with biopsy-proven IBTR were staged for locoregional and distant metastases according to current guidelines. Mastectomy was offered as standard therapy to all patients. For patients with low-risk IBTR with maximum rpT1 cN0 cM0 tumors and preferably positive hormone receptor status without overexpression of Her2 (human epidermal growth factor receptor 2) who refused modified radical mastectomy, a second BCT was considered. Multifocal tumors were not treated with a second BCT. The decision for a second BCT was further influenced by the initial therapy, the ability to obtain negative resection margins, and the possibility of preserving a reasonable cosmetic result.

Second BCT was performed via re-excision and re-irradiation of the tumor bed, using high-dose-rate (HDR brachytherapy) or pulsed-dose-rate (PDR brachytherapy) multi-catheter interstitial brachytherapy. Post-implant CT-based treatment planning was performed. For appropriate localization of the former tumor bed, preoperative clinical examination, preoperative imaging (mammograms, ultrasound, and MRI if available; $n=4$ patients), surgical reports, position of the skin scar, and surgical clips ( $n=4$ patients) were taken into consideration. Post-operative ultrasound was additionally applied for identification of the tumor cavity and post-operative tissue changes. Clinical target volume (CTV) was created by delineation of the tumor bed and non-isotropic geometrical expansion. In each direction, the safety margin was calculated by taking into account the size of the free resection margin in the respective direction. CTV contouring was based on the slightly modified recommendations by the GEC-ESTRO Breast Cancer Working Group [22]. In detail, the safety margin had to be at least $10 \mathrm{~mm}$ (in case of $\mathrm{R} 0$ resection), up to a maximum of $20 \mathrm{~mm}$ (in case of close margin resection). As treatment was performed with multi-catheter brachytherapy, no additional PTV margin was added. Dose constraints were used in line with the ESTRO-ACROP guideline [23]. A typical HDR brachytherapy treatment plan is shown in Figure 1.

Eleven patients received HDR brachytherapy and 8 patients PDR brachytherapy using ${ }^{192}$ Ir afterloading. For HDR brachytherapy, a total dose of 34.2 Gy in fractions of $3.8 \mathrm{~Gy}(n=9)$ or $32 \mathrm{~Gy}$ in fractions of $4 \mathrm{~Gy}(n=2)$ was applied in two fractions per day. PDR brachytherapy was performed one pulse per hour using a total dose of $50 \mathrm{~Gy}$ in pulses of $0.5 \mathrm{~Gy}(n=4), 49.8 \mathrm{~Gy}$ in pulses of $0.6 \mathrm{~Gy}(n=2), 50.4 \mathrm{~Gy}$ in pulses of $0.6 \mathrm{~Gy}(n=1)$ or $0.7 \mathrm{~Gy}$ $(n=1)$. A classical flexible plastic catheter technique was applied. The number of catheters ranged between 7 and 21. Minimal distance between implant and thoracic wall as well as between implant and skin had to be more than $5 \mathrm{~mm}$ and at least $1 \mathrm{~cm}$ for reducing skin toxicity, respectively. Treatment plans were optimized using treatment planning system PLATO (Nucletron, Veenendaal, The Netherlands) until 2010 and Oncentra Brachy (Nucletron, now Elekta AB, Stockholm, Sweden) afterwards. Treatment delivery was performed with microSelectron PDR 


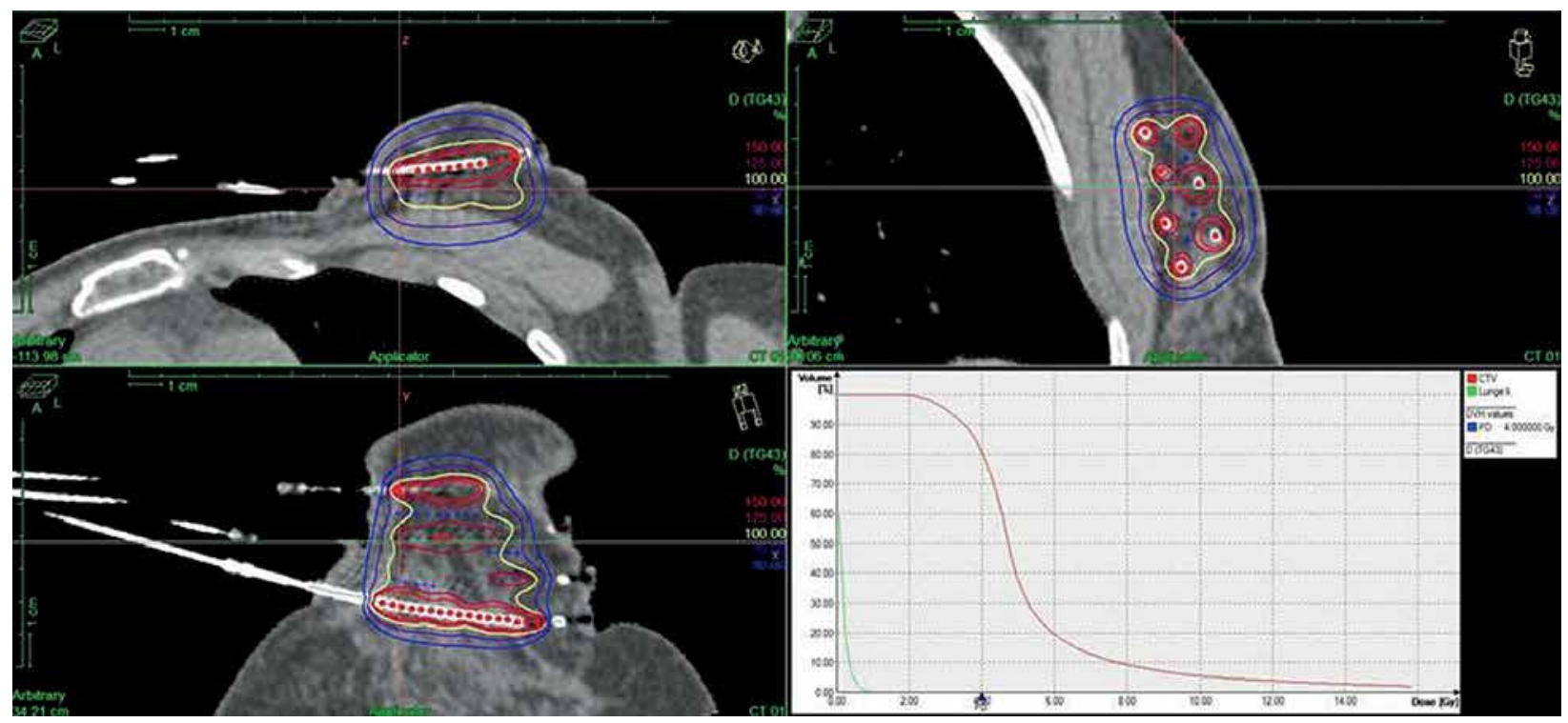

Fig. 1. Treatment plan with dose-volume histogram

or microSelectron HDR units (Nucletron, now Elekta), respectively.

Patient data were retrieved retrospectively from institutional databases in accordance with institutional ethical policies. For patients who received their initial whole breast irradiation outside of the Heidelberg University Hospital, all treatment-related documents were collected. Analyses of surgical and pathological reports as well as reviews of treatment plans were performed. Overall survival (OS) was calculated in months from the start of brachytherapy until the last date of follow-up or death. Disease-free survival (DFS) as well as local control (LC) and locoregional control (LRC) were calculated from the start of brachytherapy until the first diagnosis of recurrent disease. Local recurrence was defined as any relapse within the ipsilateral breast tissue, while regional recurrence was considered as occurrence of regional lymph node metastases (axilla, supraclavicular fossa, internal mammary chain). Recurrences at any other sites were classified as distant metastases. The data analysis was censored as not all patients suffered from an event during follow-up time. Kaplan-Meier method was used to estimate survival rates of OS, DFS, LC, and LRC. Kaplan-Meier plots were generated using a software tool SPSS 24.0. Acute toxicity was defined as occurence of side effects within 3 months following brachytherapy, while later occurence was classified as late toxicity. Toxicity was evaluated according the Common Terminology Criteria for Adverse Events (CTCAE) version 5.0. The present study was approved by the ethics committee of the Medical Faculty of Heidelberg University (S-047/2016).

\section{Results}

\section{Patient characteristics}

Patient and treatment characteristics are illustrated in Table 1. Patients' median age at the time of initial breast cancer diagnosis and of IBTR was 50.4 years and 63.7 years, respectively. Most IBTRs occurred in or close to the primary tumor site: $79 \%(n=15)$ were detected in the same quadrant, $11 \%(n=2)$ in the nearby quadrant, and another $11 \%(n=2)$ in a different quadrant of the breast. One patient was simultaneously diagnosed with contralateral breast cancer and IBTR. Primary tumors as well as IBTRs were staged pTis to pT1c, with an average tumor size of $11 \mathrm{~mm}$ and $12 \mathrm{~mm}$, respectively. For the primary tumors, histological lymph node status was documented in nearly $90 \%$ of patients. After diagnosis of the primary tumor, $63 \%(n=12)$ of patients received axillary lymph node dissection, while sentinel lymph node biopsy was performed in $26 \%(n=5)$ of cases. For $11 \%$ of patients $(n=2)$, there was no histological lymph node status documented. At the time point of IBTR, second axillary dissection was not performed in $89 \%(n=17)$ of the cases to reduce the risk of lymphedema. In three cases, histology of IBTR differed from histology of the primary tumor: two patients were diagnosed with invasive lobular carcinomas as primary tumors and invasive ductal carcinomas as IBTR, while one patient suffered from an invasive ductal carcinoma as primary tumor and was diagnosed with an invasive mucinous carcinoma as IBTR. Changes in the receptor status were detected in three other women: while the primary tumor of two patients was Her2 positive, local recurrences were Her2 negative. One woman was initially diagnosed with hormone receptor negative breast cancer, while IBTR was an estrogen and progesterone receptor-positive tumor.

\section{Second breast conserving therapy}

Median follow-up for all patients was 66 months after re-treatment of IBTR (range, 18-120 months) and 206 months after initial treatment of the primary tumor (range, 143-375 months). The median time interval between primary tumor and IBTR was 160 months (range, 27-290 months). 
Table 1. Patients, tumors, and treatments characteristics

\begin{tabular}{|c|c|c|c|c|c|c|c|}
\hline \multirow[t]{2}{*}{ Characteristic } & & \multicolumn{3}{|c|}{ Primary tumor } & \multicolumn{3}{|c|}{ IBTR } \\
\hline & & $\begin{array}{l}\text { Patients } \\
\text { (total, 19) }\end{array}$ & $\%$ & Median (range) & $\begin{array}{l}\text { Patients } \\
\text { (total, 19) }\end{array}$ & $\%$ & Median (range) \\
\hline \multicolumn{2}{|c|}{ Age at diagnosis (years) } & & & $50.4(41-71)$ & & & $63.7(55-85)$ \\
\hline \multicolumn{2}{|c|}{ Time to IBTR (months) } & & & & & & $159.8(27-290)$ \\
\hline \multirow[t]{2}{*}{ EBRT } & Dose (Gy) & 19 & & $50.4(50.4-50.4)$ & & & \\
\hline & Boost (Gy) & 16 & 84 & $11.4(8-16)$ & & & \\
\hline \multicolumn{2}{|l|}{ pT size $(\mathrm{mm})$} & & & $11(5-21)$ & & & $12(5-24)$ \\
\hline \multirow{3}{*}{$\begin{array}{l}\text { Tumor location } \\
\text { (quadrant) }\end{array}$} & Same & & & & 15 & 78 & \\
\hline & Nearby & & & & 2 & 11 & \\
\hline & Other & & & & 2 & 11 & \\
\hline \multirow[t]{5}{*}{ Histology } & Lobular & 2 & 11 & & 1 & 5 & \\
\hline & Ductal & 4 & 21 & & 10 & 53 & \\
\hline & Mucinous & 0 & 0 & & 1 & 5 & \\
\hline & DCIS & 3 & 16 & & 1 & 5 & \\
\hline & Unknown & 10 & 52 & & 6 & 32 & \\
\hline \multirow[t]{4}{*}{ pN status } & pNO & 15 & 78 & & 5 & 26 & \\
\hline & pN1a & 2 & 11 & & 0 & 0 & \\
\hline & $>$ pN1a & 0 & 0 & & 0 & 0 & \\
\hline & Unknown & 2 & 11 & & 14 & 74 & \\
\hline \multirow[t]{3}{*}{ Pathological grade } & G1 & 8 & 42 & & 5 & 26 & \\
\hline & $\mathrm{G} 2$ & 7 & 37 & & 11 & 58 & \\
\hline & G3 & 4 & 21 & & 3 & 16 & \\
\hline \multirow[t]{3}{*}{ ER/PR status } & Negative & 4 & 21 & & 3 & 16 & \\
\hline & Positive & 14 & 74 & & 16 & 84 & \\
\hline & Unknown & 1 & 5 & & 0 & 0 & \\
\hline \multirow[t]{3}{*}{ Her2 status } & Negative & 7 & 36 & & 19 & 100 & \\
\hline & Positive & 6 & 32 & & 0 & 0 & \\
\hline & Unknown & 6 & 32 & & 0 & 0 & \\
\hline \multirow[t]{2}{*}{ Endocrine therapy } & Yes & 14 & 74 & & 14 & 74 & \\
\hline & No & 5 & 26 & & 5 & 26 & \\
\hline \multirow[t]{2}{*}{ Chemotherapy } & Yes & 6 & 32 & & 2 & 11 & \\
\hline & No & 13 & 68 & & 17 & 89 & \\
\hline \multirow[t]{2}{*}{ BT } & HDR-BT & 0 & 0 & & 11 & 58 & \\
\hline & PDR-BT & 0 & 0 & & 8 & 42 & \\
\hline
\end{tabular}

IBTR - ipsilateral breast tumor recurrence, $p N$ status - pathological axillary lymph node status, ER/PR status - hormonal receptor status (negative = estrogen AND progesterone receptor negative), Her2 status - status of human epidermal growth factor receptor $2, B T$ - brachytherapy, HDR-BT and PDR-BT - high-dose-rate or pulsed-dose-rate multi-catheter interstitial brachytherapy, DCIS - ductal carcinoma in situ, pT size - pathological tumor size, EBRT - external beam radiation therapy

The Kaplan-Meier-estimated LC rate was $100 \%$ at 5 years, with only one second IBTR after 77 months (Figure 2), leading to an overall survival rate of $100 \%$ at 5 years (Figure 3). All women were still alive when the analysis was conducted. The only second IBTR occurred 164 months after the first irradiation and 77 months after re-irradiation of the initial IBTR (PDR brachytherapy). In this patient, second IBTR was located in the retroareolar area, which was the same location as the primary tumor. On the contrary, the initial IBTR was diagnosed in the upper lateral quadrant. The diagnosis of second IBTR in this patient was accompanied by the diagnosis of distant metastases includ- ing diffuse bone metastases. The patient was treated with mastectomy (R0), and palliative irradiation of bone matastases was performed along with denosumab for osteoprotection. Furthermore, endocrine therapy (fulvestrant) and chemotherapy (paclitaxel) were administered. At the end of follow-up, this patient was still alive (43 months after diagnosis of second IBTR). No further patients were diagnosed with locoregional or distant metastases.

\section{Toxicity}

Re-irradiation with brachytherapy was tolerated well with only mild acute toxicity. Only cutaneous erythema 


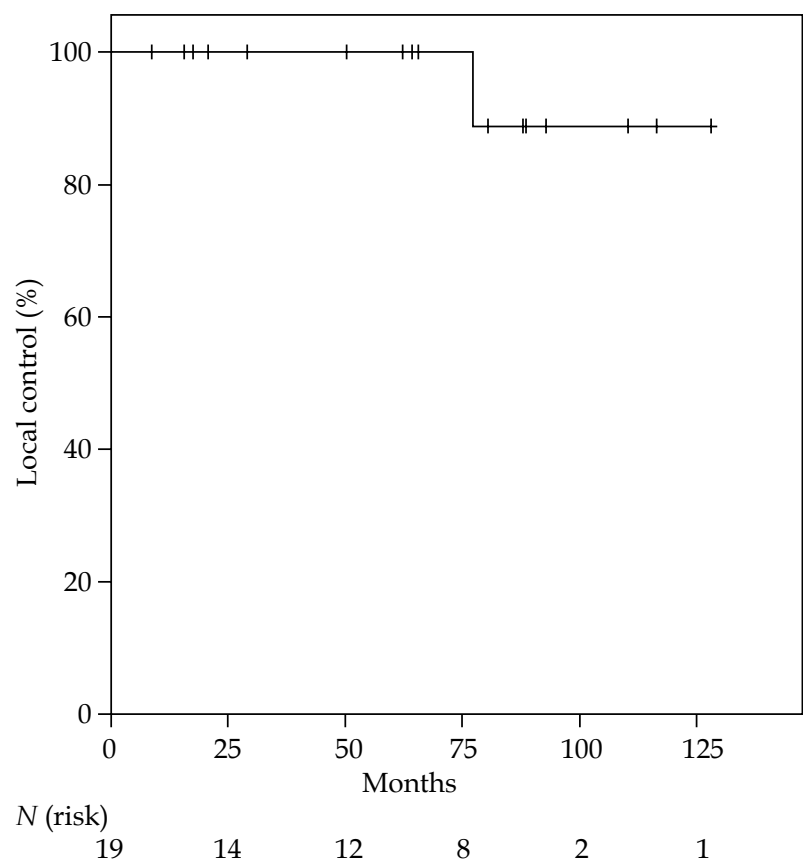

Fig. 2. Kaplan-Meier estimated local control following second BCT, including lumpectomy and partial breast re-irradiation with brachytherapy for IBRT

and dry desquamation CTCAE grade I-II were diagnosed in 9 patients, respectively. No further acute toxicities occurred. Regarding late toxicity, $63 \%$ of patients developed side effects, with fibrosis seen in $37 \%(7 / 19)$ of patients, lipid necrosis in $11 \%(2 / 19)$, hyperpigmentation in $47 \%$ (9/19), and telangiectasia in $11 \%(2 / 19)$, respectively. No patient presented with side effects $\geq$ grade 3 . Two patients suffered from fat necrosis in an area of about $3 \times 3 \mathrm{~cm}$. These two patients received equal cumulative doses as those without any side effects. One of these women was re-irradiated with HDR brachytherapy, the other with PDR brachytherapy.

\section{Discussion}

Salvage mastectomy continues to be the recommended standard therapy in case of IBTR. However, many women facing mastectomy suffer from impaired body image and diminished self-esteem, and express desire for breast preservation if possible $[24,25,26]$. After second BCT with salvage lumpectomy alone, the rate of local recurrence increased to about $20 \%[3,9,11,12]$ compared to only $10 \%$ in patients treated with salvage mastectomy $[3,10,11,12]$. In the past, there have been concerns about the safety and toxicity of a second course of breast irradiation. However, several studies now suggest that re-irradiation is feasible and safe $[3,15,16,17,18,19,27,28]$. The results of the current study are also promising and favorable compared with other analyses $[3,16,18,19,20]$. The LC rate was excellent and in the same range as expected for mastectomy with $100 \%$ at 5 years. Some minor, mainly retrospective studies also indicate that in selected low-risk patients, similar local recurrence rates to salvage

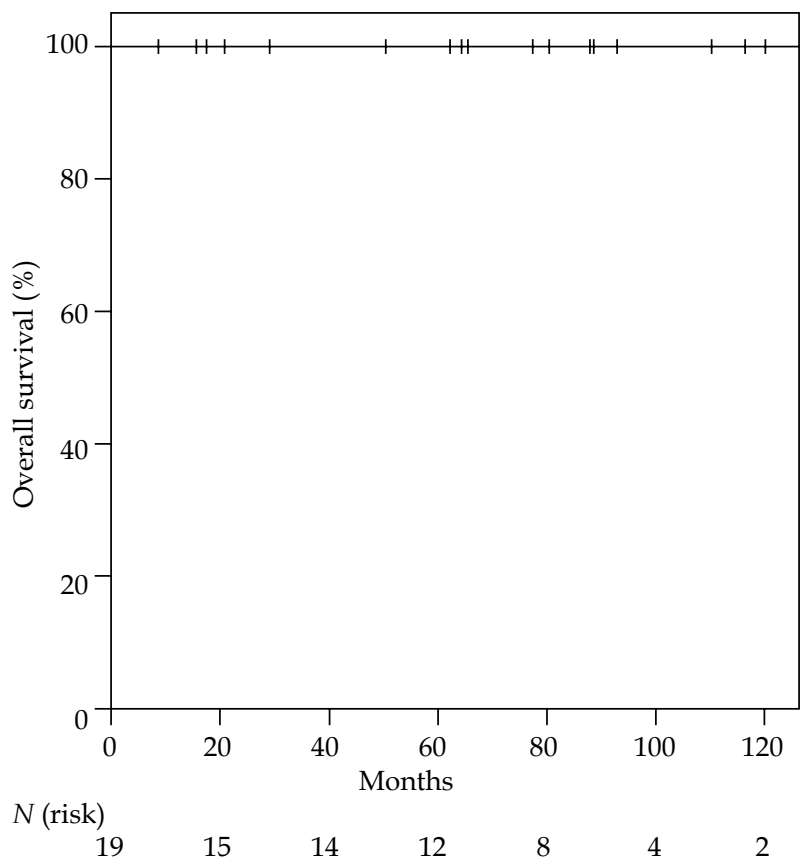

Fig. 3. Kaplan-Meier estimated overall survival following second BCT, including lumpectomy and partial breast re-irradiation with brachytherapy for IBRT

mastectomy can be detected, if adjuvant re-irradiation is added to lumpectomy $[3,14,16,17,18,19,20]$. In a retrospective analysis from 2013, Hannoun-Levi et al. reported 5- and 10-year actuarial local control rates of about $94 \%$ and $93 \%$, following lumpectomy and re-irradiation using multi-catheter brachytherapy [3], and 5-year local control rates of about $77 \%$ in an older study with less selected patients (tumor sizes pT1 to pT3) [16]. A study of Deutsch et al. from 2002 presented an estimated 5-year disease-free survival rate of only $69 \%$ after second BCT and percutaneous re-irradiation. However, in this analysis, insufficient information about appropriate patient selection was reported [14]. In another analysis of Kauer-Dorner et al., the 5 -year local control rate was $93 \%$ after re-irradiation. However, only $64 \%$ of patients were staged pT1 and only $67 \%$ pN0 in this study [20]. Notably, the slightly better results of the current study might be explained by careful patient selection, what has been validated by others [29]. Second BCT followed by partial breast irradiation was only offered to patients with early-stage low-risk recurrences with favorable tumor characteristics. As a result, the overall survival rate was $100 \%$ at 5 years in the present work, supporting BCT plus re-irradiation as an alternative treatment to mastectomy in carefully selected patients.

Additionally, salvage mastectomy is accompanied by a significant impairment of quality of life as well as emotional and physical distress [24,25,30]. After second breast-conserving therapy, patients are known to report significantly better quality of life in respect to body image, social functioning, emotional functioning as well as role functioning when compared to mastectomy [31]. Other studies even described significantly increased anxiety 
and depression levels in women treated with mastectomy compared to BCT (Hospital Anxiety and Depression Scale, 6.96 vs. 7.8 points for anxiety and 6.8 vs. 8.04 points for depression) [32]. Younger age was also shown to be an important predictor for greater impairment of quality of life following mastectomy [33]. Emotional and social functioning, and financial and future health worries were also significantly worse for younger patients undergoing mastectomy. But even patients with more than 70 years of age, reported higher body image and lifestyle scores when treated with BCT compared to mastectomy [34]. However, the diagnosis of breast cancer recurrence itself is already associated with significant changes in quality of life [35]. While partnership and family support are the predictors for good quality of life [31], family members of breast cancer patients also reported significant impairments in their own emotional well-being [36].

The time interval between primary tumor and diagnosis of IBTR is known to be an independent prognostic factor for overall survival, with a better prognosis if the time interval exceeds 36 months [37]. In the present study, the median time interval between primary tumor and IBTR was 159.8 months, with no shorter time interval than 36 months in any of the patients. However, some of the breast tumor recurrences in the current study might represent new primary tumors, as in at least 3 cases changes in histology were documented: two patients were diagnosed with invasive lobular carcinomas as primary tumors and invasive ductal carcinomas as IBTR, while one patient suffered from an invasive ductal carcinoma as primary tumor and was diagnosed with an invasive mucinous carcinoma as IBTR. Furthermore, three other women presented with changes in their receptor status: while the primary tumor of two patients was Her2 positive, the local recurrences were Her2 negative. The remaining woman was initially diagnosed with hormone receptor negative breast cancer, while IBTR was an estrogen and progesterone receptor positive tumor. However, this phenomenon is widely known in recurrent disease $[38,39]$.

The techniques for re-irradiation of the breast are numerous, including conventionally fractionated partial breast external beam re-irradiation $[27,40]$ or different techniques of intraoperative radiotherapy (IORT) [41,42]. However, the largest evidence for re-irradiation is found for multi-catheter brachytherapy [43]. For accelerated partial breast irradiation, feasibility and effectivity of this technique has already been demonstrated by others $[44,45]$, and since more than 12 years, multi-catheter brachytherapy has been applied for re-irradiation at the Heidelberg University Hospital. With this technique, side effects were rare in general, with hyperpigmentation and fibrosis representing the most frequent adverse events in the current study. Compared to treatment concepts using partial breast external beam re-irradiation, multi-catheter brachytherapy is associated with superior rates of treatment-related side effects. The NRG Oncology RTOG 1014 trial, a prospective study investigating toxicity, tolerance, and safety of partial external beam re-irradiation for inbreast recurrence, recorded treatment-related skin fibrosis and breast pain adverse events in $71 \%$ of cases [27].
Meanwhile, the treatment-related toxicity is seen in $63 \%$ of patients following multi-catheter brachytherapy in the present study. The slightly higher rate of skin toxicity in the NRG Oncology RTOG1014 trial may be explained by higher skin doses during external beam irradiation. Promising preliminary treatment outcomes of this study have been presented at 2016 Astro Annual Meeting, supporting second breast conservation as an alternative to mastectomy [46].

In the present analyzed patient cohort, no pulmonary problems, rib fractures, or second malignancies have been reported so far. However, the percentage of late side effects may increase with a longer follow-up. While in the present cohort, late side effects in general were similar or lower than in the literature [20,47]; $47 \%$ of patients had hyperpigmentation compared to reported rates of $9 \%$ to $29 \%$ in the literature $[19,20,47]$. This may be explained by the retrospective character of the present work. Hyperpigmentation due to the primary irradiation was not taken into account, as baseline hyperpigmentation has not been documented. Reported rates of fat necrosis after interstitial brachytherapy of about $20 \%$, as observed by Budrukkar et al. [48], could not be confirmed in our study as fat necrosis was noticed only in $10 \%$ of patients.

Limitations in the current work are mainly due to the retrospective character of the analysis. Furthermore, due to careful patient selection and a low a priori risk of IBTR after first BCT, the present single center cohort of 10 -year experience in partial breast re-irradiation is rather small. Although, 10-year experiences are already scarce in the application of modern techniques, an even longer follow-up of 10 to 20 years would be needed for sufficient evidence. A precise differentiation between true local recurrences and new primaries arising in the treated breast was not possible. Long interval between first BCT and IBTR as well as differences in histology suggest that some patients included in this analysis might have had new primaries. This may have positively influenced the oncological outcome observed in this analysis [49]. Nevertheless, this study provides encouraging data for the feasibility, safety, and efficacy of a second BCT with lumpectomy followed by partial breast re-irradiation in case of IBTR. For highly selected patients with low-risk recurrences, this treatment approach allows avoiding salvage mastectomy, which is often accompanied by severe impairments in quality of life and body image. Our data confirm that a repeated course of irradiation following second lumpectomy due to IBTR appears to be a reasonable alternative to mastectomy, at least for some patients. This is supported by current national and international guidelines recommending that individualized treatment strategies including second BCT should be considered and discussed by multidisciplinary tumor boards $[3,43,50]$. Randomized controlled trials comparing salvage mastectomy to second BCT including re-irradiation are urgently needed for an well-founded assessment whether mastectomy can be avoided for selected patients in the future [50]. However, conducting such a trial may be futile due to patients' preferences regarding randomization between second BCT and mastectomy. 


\section{Conclusions}

The current study demonstrates that in patients with small, low-risk local breast tumor recurrences, local control rates after second BCT with salvage lumpectomy and partial breast re-irradiation are comparable to those after mastectomy. This analysis provides encouraging data for the feasibility, safety, and efficacy of a second BCT. Our results may support a rational discussion about the opportunity of second BCT followed by partial breast irradiation for selected low-risk patients.

\section{Funding section}

The resources and facilities of the Medical Faculty of Heidelberg University were used in conducting this study. There was no additional source of funding.

\section{Ethic approval and consent to participate}

The analysis was approved by the Ethics committee of Heidelberg University (S-047/2016). Written informed consent for publication of their images was obtained from the respective patients.

\section{Disclosure}

The authors report no conflict of interest.

\section{References}

1. Breast cancer incidence, mortality and prevalence worldwide in 2012. http://globocan.iarc.fr/old/FactSheets/cancers/ breast-new.asp (access online 22/08/2018).

2. Brewster AM, Hortobagyi GN, Broglio KR et al. Residual risk of breast cancer recurrence 5 years after adjuvant therapy. J Natl Cancer Inst 2008; 100: 1179-1183.

3. Hannoun-Levi JM, Resch A, Gal J et al. Accelerated partial breast irradiation with interstitial brachytherapy as second conservative treatment for ipsilateral breast tumour recurrence: multicentric study of the GEC-ESTRO Breast Cancer Working Group. Radiother Oncol 2013; 108: 226-231.

4. Smith BD, Arthur DW, Buchholz TA et al. Accelerated partial breast irradiation consensus statement from the American Society for Radiation Oncology (ASTRO). J Am Coll Surg 2009; 209: 269-277.

5. Early Breast Cancer Trialists' Collaborative Group, Darby $\mathrm{S}$, McGale $\mathrm{P}$, Correa $\mathrm{C}$ et al. Effect of radiotherapy after breast-conserving surgery on 10-year recurrence and 15-year breast cancer death: meta-analysis of individual patient data for 10,801 women in 17 randomised trials. Lancet 2011; 378: $1707-1716$

6. Kunkler IH, Williams LJ, Jack WJ et al. Breast-conserving surgery with or without irradiation in women aged 65 years or older with early breast cancer (PRIME II): a randomised controlled trial. Lancet Oncol 2015; 16: 266-273.

7. Veronesi U, Saccozzi R, Del Vecchio M, et al. Comparing radical mastectomy with quadrantectomy, axillary dissection, and radiotherapy in patients with small cancers of the breast. N Engl J Med 1981; 305: 6-11.

8. Fontes KP, Veiga DF, Naldoni AC et al. Physical activity, functional ability, and quality of life after breast cancer surgery. J Plast Reconstr Aesthet Surg 2019; 72: 394-400.

9. Kurtz JM, Amalric R, Brandone $\mathrm{H}$ et al. Results of wide excision for mammary recurrence after breast-conserving therapy. Cancer 1988; 61: 1969-1972.
10. Kurtz JM, Jacquemier J, Amalric R et al. Is breast conservation after local recurrence feasible? Eur J Cancer 1991; 27: 240-244.

11. Abner AL, Recht A, Eberlein T et al. Prognosis following salvage mastectomy for recurrence in the breast after conservative surgery and radiation therapy for early-stage breast cancer. J Clin Oncol 1993; 11: 44-48.

12. Alpert TE, Kuerer HM, Arthur DW et al. Ipsilateral breast tumor recurrence after breast conservation therapy: outcomes of salvage mastectomy vs. salvage breast-conserving surgery and prognostic factors for salvage breast preservation. Int J Radiat Oncol Biol Phys 2005; 63: 845-851.

13. Ishitobi M, Komoike $Y$, Nakahara S et al. Repeat lumpectomy for ipsilateral breast tumor recurrence after breast-conserving treatment. Oncology 2011; 81: 381-386.

14. Deutsch M. Repeat high-dose external beam irradiation for inbreast tumor recurrence after previous lumpectomy and whole breast irradiation. Int J Radiat Oncol Biol Phys 2002; 53: 687-691.

15. Resch A, Fellner C, Mock U et al. Locally recurrent breast cancer: pulse dose rate brachytherapy for repeat irradiation following lumpectomy - a second chance to preserve the breast. Radiology 2002; 225: 713-718.

16. Hannoun-Levi JM, Houvenaeghel G, Ellis S et al. Partial breast irradiation as second conservative treatment for local breast cancer recurrence. Int J Radiat Oncol Biol Phys 2004; 60: 1385-1392.

17. Kraus-Tiefenbacher U, Bauer L, Scheda A et al. Intraoperative radiotherapy (IORT) is an option for patients with localized breast recurrences after previous external-beam radiotherapy. BMC Cancer 2007; 7: 178.

18. Guix B, Lejárcegui JA, Tello JI et al. Exeresis and brachytherapy as salvage treatment for local recurrence after conservative treatment for breast cancer: results of a ten-year pilot study. Int J Radiat Oncol Biol Phys 2010; 78: 804-810.

19. Hannoun-Levi JM, Castelli J, Plesu A et al. Second conservative treatment for ipsilateral breast cancer recurrence using high-dose rate interstitial brachytherapy: preliminary clinical results and evaluation of patient satisfaction. Brachytherapy 2011; 10: 171-177.

20. Kauer-Dorner D, Pötter R, Resch A et al. Partial breast irradiation for locally recurrent breast cancer within a second breast conserving treatment: alternative to mastectomy? Results from a prospective trial. Radiother Oncol 2012; 102: 96-101.

21. Polgár C, Major T, Sulyok Z, et al. Second breast-conserving surgery and reirradiation with interstitial high-dose-rate brachytherapy for the management of intra-breast recurrences - 5-year results. Magy Onkol 2012; 56: 68-74.

22. Major T, Gutiérrez C, Guix B et al. Recommendations from GEC ESTRO Breast Cancer Working Group (II): Target definition and target delineation for accelerated or boost partial breast irradiation using multicatheter interstitial brachytherapy after breast conserving open cavity surgery. Radiother Oncol 2016; 118: 199-204.

23. Strnad V, Major T, Polgar C et al. ESTRO-ACROP guideline: Interstitial multi-catheter breast brachytherapy as Accelerated Partial Breast Irradiation alone or as boost - GEC-ESTRO Breast Cancer Working Group practical recommendations. Radiother Oncol 2018; 128: 411-420.

24. Ganz PA, Schag AC, Lee JJ et al. Breast conservation versus mastectomy. Is there a difference in psychological adjustment or quality of life in the year after surgery? Cancer 1992; 69: 1729-1738.

25. Rowland JH, Desmond KA, Meyerowitz BE et al. Role of breast reconstructive surgery in physical and emotional outcomes among breast cancer survivors. J Natl Cancer Inst 2000; 92: 1422-1429. 
26. Maunsell E, Brisson J, Deschênes L et al. Randomized trial of a psychologic distress screening program after breast cancer: effects on quality of life. J Clin Oncol 1996; 14: 2747-2755.

27. Arthur DW, Winter KA, Kuerer HM et al. NRG Oncology-radiation therapy oncology group study 1014: 1-year toxicity report from a phase 2 study of repeat breast-preserving surgery and 3-dimensional conformal partial-breast reirradiation for in-breast recurrence. Int J Radiat Oncol Biol Phys 2017; 98: 1028-1035.

28. Niehoff P, Dietrich J, Ostertag H et al. High-dose-rate (HDR) or pulsed-dose-rate (PDR) perioperative interstitial intensity-modulated brachytherapy (IMBT) for local recurrences of previously irradiated breast or thoracic wall following breast cancer. Strahlenther Onkol 2006; 182: 102-107.

29. Montagne L, Gal J, Chand ME et al. GEC-ESTRO APBI classification as a decision-making tool for the management of 2 nd ipsilateral breast tumor event. Breast Cancer Res Treat 2019; 176: 149-157.

30. Dorval M, Maunsell E, Deschênes L et al. Type of mastectomy and quality of life for long term breast carcinoma survivors. Cancer 1998; 83: 2130-2138.

31. Jendrian S, Steffens K, Schmalfeldt B et al. Quality of life in patients with recurrent breast cancer after second breast-conserving therapy in comparison with mastectomy: the German experience. Breast Cancer Res Treat 2017; 163: 517-526.

32. Kaminska M, Kubiatowski T, Ciszewski T et al. Evaluation of symptoms of anxiety and depression in women with breast cancer after breast amputation or conservation treated with adjuvant chemotherapy. Ann Agric Environ Med 2015; 22: 185-189.

33. Costanzo ES, Lutgendorf SK, Mattes ML et al. Adjusting to life after treatment: distress and quality of life following treatment for breast cancer. Br J Cancer 2007; 97: 1625-1631.

34. Engel J, Kerr J, Schlesinger-Raab A et al. Quality of life following breast-conserving therapy or mastectomy: results of a 5-year prospective study. Breast J 2004; 10: 223-231.

35. Bull AA, Meyerowitz BE, Hart S et al. Quality of life in women with recurrent breast cancer. Breast Cancer Res Treat 1999; 54: 47-57.

36. Northouse LL, Mood D, Kershaw T et al. Quality of life of women with recurrent breast cancer and their family members. J Clin Oncol 2002; 20: 4050-4064.

37. Anderson SJ, Wapnir I, Dignam JJ et al. Prognosis after ipsilateral breast tumor recurrence and locoregional recurrences in patients treated by breast-conserving therapy in five $\mathrm{Na}$ tional Surgical Adjuvant Breast and Bowel Project protocols of node-negative breast cancer. J Clin Oncol 2009; 27: 24662473.

38. Ilgun S, Sarsenov D, Erdogan Z et al. Receptor discordance rate and its effects on survival in primary and recurrent breast cancer patients. J BUON 2016; 21: 1425-1432.

39. Zhu YY, Si W, Ji TF et al. The variation and clinical significance of hormone receptors and Her-2 status from primary to metastatic lesions in breast cancer patients. Tumour Biol 2016; 37: 7675-7684.

40. Janssen S, Rades D, Meyer A et al. Local recurrence of breast cancer: conventionally fractionated partial external beam re-irradiation with curative intention. Strahlenther Onkol 2018; 194: 806-814.

41. Veronesi U, Orecchia R, Maisonneuve P et al. Intraoperative radiotherapy versus external radiotherapy for early breast cancer (ELIOT): a randomised controlled equivalence trial. Lancet Oncol 2013; 14: 1269-1277.

42. Vaidya JS, Joseph DJ, Tobias JS et al. Targeted intraoperative radiotherapy versus whole breast radiotherapy for breast cancer (TARGIT-A trial): an international, prospective, ran- domised, non-inferiority phase 3 trial. Lancet 2010; 376: 91 102.

43. Harms W, Budach W, Dunst J et al. DEGRO practical guidelines for radiotherapy of breast cancer VI: therapy of locoregional breast cancer recurrences. Strahlenther Onkol 2016; 192: 199-208.

44. Latorre JA, Galdós P, Buznego LA et al. Accelerated partial breast irradiation in a single 18 Gy fraction with highdose-rate brachytherapy: preliminary results. J Contemp Brachytherapy 2018; 10: 58-63.

45. Cozzi S, Laplana M, Najjari D et al. Advantages of intraoperative implant for interstitial brachytherapy for accelerated partial breast irradiation either frail patients with early-stage disease or in locally recurrent breast cancer. J Contemp Brachytherapy 2018; 10: 97-104.

46. Arthur DW, Kuerer HM, Moughan J et al. NRG Oncology/ RTOG 1014: 3 year efficacy report from a phase II study of repeat breast preserving surgery and $3 \mathrm{~d}$ conformal Partial Breast re-Irradiation (PBrI) for in-breast recurrence. Int J Radiat Oncol Biol Phys 2016; 96: 941.

47. Chadha M, Feldman S, Boolbol S et al. The feasibility of a second lumpectomy and breast brachytherapy for localized cancer in a breast previously treated with lumpectomy and radiation therapy for breast cancer. Brachytherapy 2008; 7: 22-28.

48. Budrukkar A, Jagtap V, Kembhavi $S$ et al. Fat necrosis in women with early-stage breast cancer treated with accelerated partial breast irradiation (APBI) using interstitial brachytherapy. Radiother Oncol 2012; 103: 161-165.

49. Komoike Y, Akiyama F, Iino Y et al. Analysis of ipsilateral breast tumor recurrences after breast-conserving treatment based on the classification of true recurrences and new primary tumors. Breast Cancer 2005; 12: 104-111.

50. Hannoun-Levi JM, Ihrai T, Courdi A. Local treatment options for ipsilateral breast tumour recurrence. Cancer Treat Rev 2013; 39: 737-741. 